\title{
CHITOSAN POLYMER AS A COAT OF CALCIUM ALGINATE MICROCAPSULES LOADED BY NON-STEROIDAL ANTI- INFLAMMATORY DRUG
}

\author{
S. Abd El-Rasoul and Mahmoud M. Ahmed*
}

Department of Pharmaceutics and Industrial Pharmacy, Faculty of Pharmacy, Al-Azhar University, Assiut branch, Assiut, Egypt

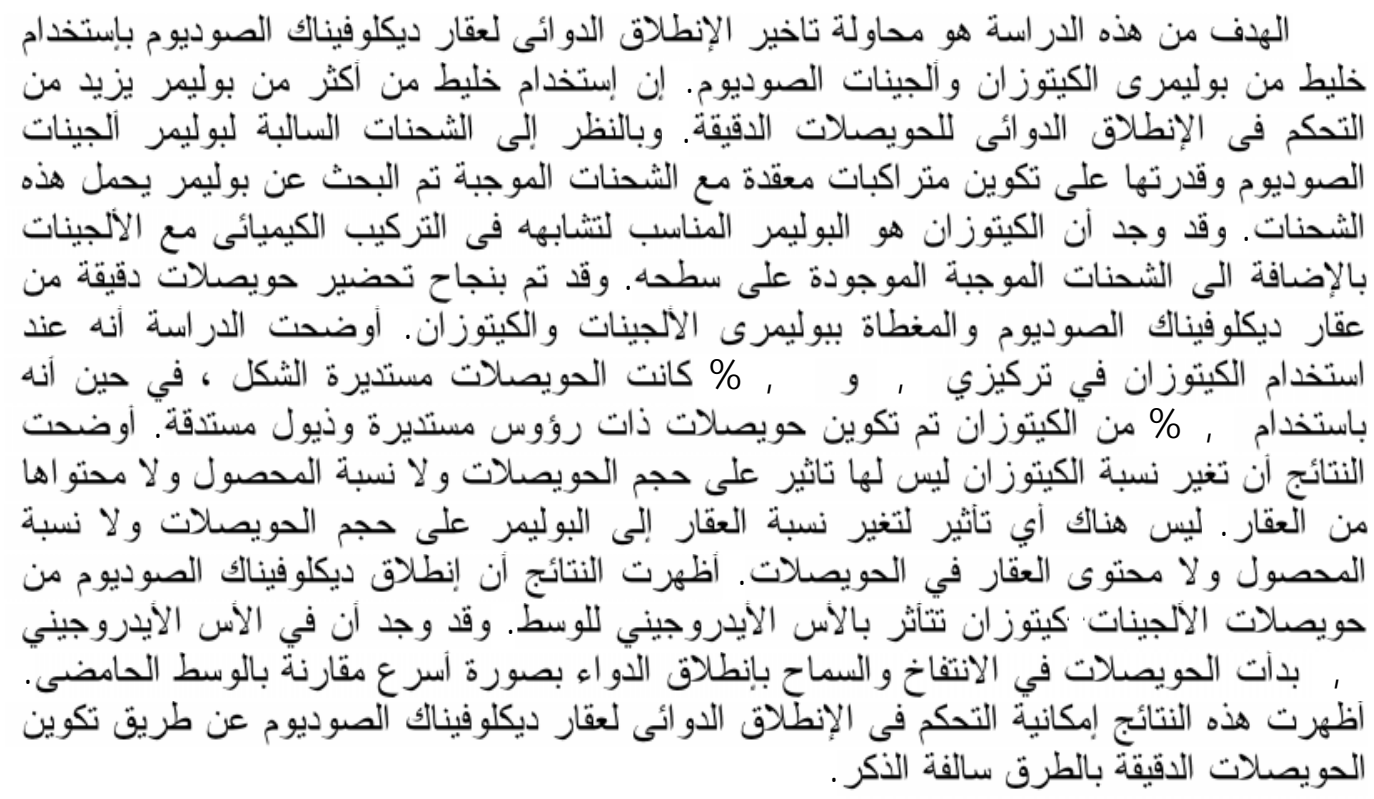

In a trial to delay the release rate of diclofenac sodium from alginate coated microcapsules, the use of a copolymer was suggested. Mixtures of polymers can have a significant properties than that of individual polymer to achieve sustained release microcapsules. On considering the negative charge of alginate and its ability to form polyionic complexes with a lowered tendency of erosion at higher $\mathrm{pH}$ value, a cationic polymer was seeked. Chitosan (CS) was the one of choice due to the similarity of the saccharide structures of chitosan and alginate that offers greater interaction between the two polymers and stronger inter-chains reactions relative to that between alginate and branched polymers such as polylysine. The microcapsules prepared using 0.1 and $0.25 \%$ (CS) were spherical in shape while $0.4 \%$ (CS) formed microcapsules having rounded heads and tapered tails. The change in chitosan concentration had a non-significant effect on the particle size, the yield and the drug loading efficiency. The releaser ate of diclofenac sodium from microspheres showed a $\mathrm{pH}$ dependent profile and was affected by chitosan coating. The release rate of diclofenac sodium from chitosan coated alginate microcapsules at $\mathrm{pH} 7.4$ was found to be faster than its release at pH 1.2. These results suggest this coating method to protect diclofenac sodium under acidic conditions and to permit a complete but controlled release of diclofenac sodium.

\section{INTRODUCTION}

Diclofenac sodium is an effective antiinflammatory and analgesic drug in clinical practice. Diclofenac sodium inhibits both isoforms of cyclo-oxygenase enzyme. The inhibition of cyclo-oxygenase enzyme COX-I results in a number of undesirable effects such as gastro-intestinal complaints. Moreover, diclofenac sodium has a short plasma-half-life (about 1-2 hrs) that necessitates three-times a day administration of the $\operatorname{drug}^{1}$. Therefore,

Received in 2/11/2010 \& Accepted in 9/12/2010 
diclofenac sodium is highly recommended to be prescribed in sustained release form. Chitosan, a polysaccharide derived from chitin by alkaline deacetylation, has been used to reinforce alginate microspheres based on the electrostatic interaction between carboxylate alginate groups and ammonium chitosan groups $^{2}$. Chitosan-alginate complex erode slowly in phosphate buffer, and this behavior leads to suppression of the initial release of drugs occurring for uncoated microspheres ${ }^{2}$. Usually these microspheres are produced by a two stage method, where preformed alginate microspheres are recovered and subsequently coated with chitosan ${ }^{3}$. Chitosan coating of lowsizing alginate microspheres is an awkward technique and the possibility of doing it during procedure by a simple method can be a valid alternative ${ }^{4}$. Furthermore, it would contribute to retain encapsulant during emulsification, decrease its loss during microspheres recovery, and may provide a sustained release effect ${ }^{5}$. Hence, the aim of this work was to formulate diclofenac sodium in controlled release oral dosage form. The prepared microcapsules were evaluated for their morphology and surface structure, average particle size, yield, drug loading efficiency, and drug release characteristics.

\section{EXPERIMENTAL}

\section{Materials}

Diclofenac sodium (DS): El-Nasr, Pharm. Chem. Co. (Cairo, Egypt). Sodium alginate (NaA): Sigma Chemical Co., St. Louis (USA). Calcium chloride $\left(\mathrm{CaCl}_{2}\right)$ : Adwia, El-Nasr, Pharm. Chem. Co. (Cairo, Egypt). Chitosan C2646 (CS), minimum 85\% deacetylated: Sigma Chemical Co., St. Louis (USA). Methylene chloride, Potassium dihydrogen orthephosphate, and acetic acid: Adwia, El-Nasr Pharm. Chem. Co. (Egypt). All the above materials were in analytical grade and were used without further purification.

\section{Design of the experiment}

Table 1 shows the different formulations of diclofenac sodium microcapsules. The microcapsules were prepared using drug to polymer (sodium alginate) ratios of 1:1 and 1:2 and using chitosan (CS) at three different concentrations, namely, $0.1,0.25$ and $0.4 \%$.
Sodium alginate was used at concentrations of 2 and $4 \%$ and at $0.1 \mathrm{M} \mathrm{CaCl}_{2}{ }^{6}$.

Table 1: Design of the different formulations of diclofenac sodium microcapsules prepared using sodium alginatechitosan.

\begin{tabular}{|c|c|c|c|c|}
\hline $\begin{array}{c}\text { Formulati } \\
\text { on number }\end{array}$ & $\begin{array}{c}\mathrm{CS} \\
(\%)\end{array}$ & $\begin{array}{c}\mathrm{D}: \mathrm{P}(\mathrm{NaA}) \\
\text { Ratio }\end{array}$ & $\begin{array}{c}\mathrm{NaA} \\
(\%)\end{array}$ & $\begin{array}{c}\mathrm{CaCl}_{2} \\
(\mathrm{M})\end{array}$ \\
\hline 1 & 0.10 & $1: 1$ & 2.0 & 0.1 \\
\hline 2 & 0.10 & $1: 2$ & 4.0 & 0.1 \\
\hline 3 & 0.25 & $1: 1$ & 2.0 & 0.1 \\
\hline 4 & 0.25 & $1: 2$ & 4.0 & 0.1 \\
\hline 5 & 0.40 & $1: 1$ & 2.0 & 0.1 \\
\hline 6 & 0.40 & $1: 2$ & 4.0 & 0.1 \\
\hline
\end{tabular}

\section{Preparation of alginate coated micro- capsules}

Six formulations were prepared using the different process variables as follows: Sodium alginate $(140,280 \mathrm{mg})$ was dissolved in $7 \mathrm{ml}$ distilled water in $10 \mathrm{ml}$ beaker with agitation using a magnetic stirrer, and then diclofenac sodium (140 mg) was dispersed in this solution to prepare $1: 1$ and 1:2 drug to polymer ratio respectively. Stirring continued for $10 \mathrm{~min}$. Chitosan solutions, $0.1,0.25$ or $0.4 \%$ (w/v), were prepared by the addition of chitosan to 50 $\mathrm{ml}$ distilled water containing $1 \%$ (v/v) acetic acid and left for maceration overnight. The required amount of $\mathrm{CaCl}_{2}$ was added to chitosan solution to obtain a $0.1 \mathrm{M}$ solution. Using a $10 \mathrm{ml}$ syringe, the alginate-drug suspension was added to the previous solution, dropwise, at a rate of $1 \mathrm{ml} / \mathrm{min}$ with slight stirring using a magnetic stirrer at ambient temperature. The mixture was then stirred slowly for $6 \mathrm{~min}$. to cure the prepared microcapsules. The microcapsules were separated by filtration, washed with distilled water, dried for $48 \mathrm{hrs}$ at ambient conditions and stored in desiccator until starting experiment.

\section{Microscopic examination of microcapsules}

On a suitable slide, amount of the dried microcapsules was observed under an optical microscope and microscope photographs were taken at magnification powers 40 and 100x. 


\section{Determination of the particle size of the microcapsules}

The dried microcapsules were weighed and sized using USP standard sieve set. The fraction of microcapsules remaining on each sieve was collected, and the mean particle size of the microcapsules was assigned as the percentage of microcapsules retained at each sieve multiplied by the average particle size of this sieve.

\section{Determination of the microcapsules yield}

The yield of the microcapsules was determined by dividing the weight of the prepared microcapsules by the original amount of the polymer and drug used and the results were expressed as a percentage.

\section{Determination of the diclofenac sodium content in microcapsules}

Ten milligrams of the microcapsules were added to $25 \mathrm{ml}$ phosphate buffer (pH 7.4) in a $25 \mathrm{ml}$ volumetric flask and left over night. The withdrawn samples were properly diluted and measured spectrophotometrically at $\lambda_{\max } 277$ $\mathrm{nm}$ against phosphate buffer $\mathrm{pH} 7.4$ as a blank. The experiment was done in triplicate.

\section{Release of the drug from the microcapsules}

Dissolution testing of the prepared microcapsules equivalent to $100 \mathrm{mg}$ diclofenac sodium (DS) was performed with the rotating paddle apparatus. The operating conditions were: Paddle spead of $50 \mathrm{rpm}$ and a temperature of $37^{\circ} \mathrm{C} \pm 0.5$. Regarding the dissolution medium the $\mathrm{pH}$ shift method ${ }^{7}$ was used as follows: A volume of $900 \mathrm{ml}$ of simulated gastric fluid, $\mathrm{pH} \mathrm{1.2,} \mathrm{was} \mathrm{used} \mathrm{as} \mathrm{the}$ release medium for two hours, followed by the addition of $(8.5-12 \mathrm{ml})$ of $1 \mathrm{M} \quad \mathrm{KH}_{2} \mathrm{PO}_{4}$ containing $16.75 \%(\mathrm{w} / \mathrm{v}) \mathrm{NaOH}$, in order to change the $\mathrm{pH}$ of the medium to $7.4^{7}$. Drug release was continued in this medium for six hours. Filtered samples, $3 \mathrm{ml}$ each, were removed at specified intervals throughout the whole 8 hrs. The samples were diluted appropriately with the same dissolution medium, and absorbance was measured at the predetermined $\lambda_{\max }$ of each medium against a blank of this medium. The withdrawn samples were replaced with equal volumes of the same dissolution medium and at the same temperature.

\section{Statistical analysis}

Analysis of variance (ANOVA) and t-test were used in this work. The term analysis of variance is a source of confusion. In spite of its name, ANOVA is concerned with differences between means of groups, not differences between variances. The name analysis of variance comes from the way the procedure uses variances to decide whether the means are different $^{8}$. The way it works is simple: the program looks to see what the variation (variance) is within the groups, then works out how that variation would translate into variation (i.e. differences) between the groups, taking into account how many subjects there are in the groups. If the observed differences are a lot bigger than what you'd expect by chance, you have statistical significance. t-tests are just a special case of ANOVA, if we analyze the means of two groups by ANOVA, you get the same results as doing it with a $t$ test.

\section{RESULTS AND DISCUSSION}

\section{Microcapsule morphology}

The microcapsules prepared with alginate/chitosan copolymers were examined using the light microscope. Figure 1 shows that the prepared microcapsules were almost spherical in shape at both chitosan concentrations of 0.1 and $0.25 \%$. The samples prepared using $0.4 \%$ CS concentration was formed of microcapsules having rounded heads and tapered tails and showed a tendency for agglomeration. The formed agglomerates were easily separated by slight increase of the stirring rate during the preparation process. All

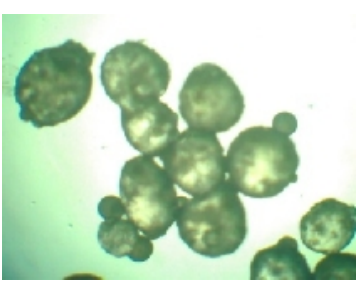

(a)

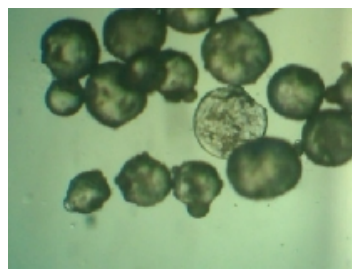

(b)
Fig. 1: Optical micrographs of the prepared microcapsules at D:P (sodium alginate) ratio $1: 1$ using (a) $0.1 \%$ chitosan and (b) $0.25 \%$ chitosan. 
the microcapsules maintained their shapes after drying. It was assumed that highly concentrated solutions of CS made the dropping process difficult and thus spherical microcapsules could not be formed. The agglomeration was attributed to the adhesive properties of chitosan.

A similar effect on the microcapsule shape was stated for nicardipine hydrochloride microcapsules at CS concentrations above $0.4 \%$, for alginate-chitosan coated microcapsules containing nicardipine at CS concentration of $0.3 \%$. The authors reported that the microcapsules agglomerated permanently in $\mathrm{CaCl}_{2} / \mathrm{CS}$ solution and that their separation was impossible 9 . For this reason, they employed chitosan at concentrations less than $0.25 \%$ $(w / v)$. On the other hand, some workers stated that alginate-chitosan coated microcapsules were almost spherical at CS concentration range of 0.2 to $1.0 \%$ while, others, found that the formed microcapsules were non-spherical on adding CS at both concentrations of 0.1 and $0.4 \%{ }^{9 \& 10}$.

\section{The particle size of the microcapsules}

The particle size analysis of alginatechitosan coated microcapsules was done using sieve analysis procedure. Figure 2 shows the mean particle size values $( \pm \mathrm{SD})$ for the microcapsules prepared by incubation for 6 min. at ambient conditions, formulations number 1 to 6 , were $743 \pm$ (49.1), $822 \pm$ (42.6), $735 \pm$ (36.9), $782 \pm$ (37.2), $785 \pm$ (47.9), $802 \pm$ (46.2) $\mu \mathrm{m}$ respectively.

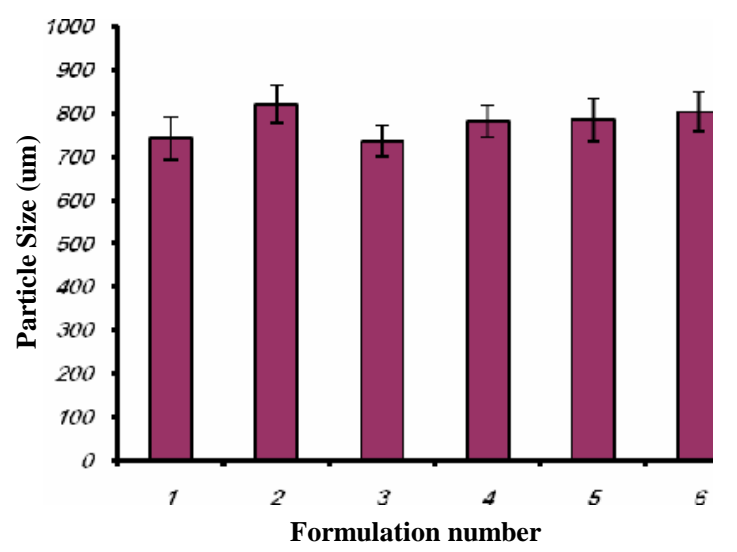

Fig. 2: Particle size of different formulae of chitosan coated microcapsules.

Drug to polymer ratio markedly affected the microcapsule size at the three different chitosan concentrations. The mean particle size of the prepared microcapsules increased with increasing the amount of the polymer (sodium alginate). The addition of CS did not significantly change the particle sizes of the formulations in comparison to that of the alginate-coated microcapsules ${ }^{6}$. This was similar to the reported results for nicardipine hydrochloride microcapsules 9 . On the other hand, other workers stated that chitosan treated alginate microcapsules were larger than alginate microcapsules ${ }^{3}$. This was attributed to extra coating. No clear relationship was found between the particle size of the microcapsules and the change in chitosan concentration.

\section{Microcapsule yield}

The percentage yield for the six prepared formulations by incubation for $6 \mathrm{~min}$. at ambient temperature was 92.9, 93.4, 93.2, 95.1, 92.2 and $91.5 \%$, respectively (Table 2). There was no obvious difference between the yield of different formulations on varying both the drug to polymer (sodium alginate) ratio and the chitosan concentration.

\section{Microcapsule content}

The data obtained in case of sodium alginate-chitosan microcapsules are listed in table 2. The actual drug loading values for formulations number 1 to 6 were 47.22, 30.92, $48.41,31.29,47.11$ and $30.54 \%$, respectively. According to drug to polymer (sodium alginate) ratio, the calculated theoretical drug loading value for formulations number 1,3 and 5 was $50 \%$ and that for formulations number 2 , 4 and 6 was $33.33 \%$. Thus, the loading efficiency values $( \pm \mathrm{SD})$ for the six formulations were computed as $94.44 \pm$ (5.47), $92.76 \pm$ (4.29), $96.82 \pm$ (6.42), $93.88 \pm$ (4.22), $94.22 \pm$ (5.57) and $91.63 \pm(4.57) \%$. The effect of changing the drug to polymer ratio on the drug loading efficiency was investigated, at each chitosan concentration, using a t-test. The tvalue was calculated to be $0.785,0.452$ and 0.811 in case of chitosan concentrations of 0.1 , 0.25 and $0.4 \%$, respectively, compared to a tabulated t-value of 2.78. It is clear that, on the contrary to the result of the calcium alginate microcapsules ${ }^{6}$, the $\mathrm{D}: \mathrm{P}$ ratio in case of alginate-chitosan coated microcapsules had no significant effect on the drug loading efficiency. 
Table 2: Production yield and drug loading efficiency of diclofenac sodium microcapsules prepared using sodium alginate-chitosan by incubation for $6 \mathrm{~min}$. at ambient conditions.

\begin{tabular}{|c|c|c|c|c|}
\hline $\begin{array}{c}\text { Formulation } \\
\text { number }\end{array}$ & $\begin{array}{c}\text { Yield } \\
(\%)\end{array}$ & $\begin{array}{c}\text { Theoretical } \\
\text { drug loading } \\
(\%)\end{array}$ & $\begin{array}{c}\text { Actual drug } \\
\text { loading } \\
(\%)\end{array}$ & $\begin{array}{c}\text { Loading efficiency } \\
(\%) \\
\text { Mean } \pm \text { SD }\end{array}$ \\
\hline 1 & $92.9 \pm 6.02$ & 50.00 & 47.22 & $94.44 \pm 5.47$ \\
\hline 2 & $93.4 \pm 5.44$ & 33.33 & 30.92 & $92.76 \pm 4.29$ \\
\hline 3 & $93.2 \pm 4.32$ & 50.00 & 48.41 & $96.82 \pm 6.42$ \\
\hline 4 & $95.1 \pm 5.56$ & 33.33 & 31.29 & $93.88 \pm 4.22$ \\
\hline 5 & $92.2 \pm 6.48$ & 50.00 & 47.11 & $94.22 \pm 5.57$ \\
\hline 6 & $91.50 \pm 7.21$ & 33.33 & 30.54 & $91.63 \pm 4.57$ \\
\hline
\end{tabular}

The chitosan concentration effect was studied at each drug to polymer ratio applying an ANOVA test (Table 3). The calculated F values were 0.386 and 0.78 on applying the test on formulations number 1,3 and 5 (prepared at D: $\mathrm{P}$ ratio of 1:1) and on formulations number 2, 4 and 6 (prepared at $\mathrm{D}: \mathrm{P}$ ratio of 1:2), respectively. The change of the CS concentration did not significantly change the loading efficiency.

\section{In-vitro release of diclofenac sodium microcapsules}

Drug release from alginate-chitosan coated microcapsules are shown in figures 3 and 4 . The time for $50 \%$ of the drug to be released $\left(\mathrm{t}_{50 \%}\right)$ was used to study the effect of changing both the drug to polymer ratio and the chitosan concentration on the release profile of diclofenac sodium from the alginate-chitosan coated microcapsules. For the D: P ratio effect, a t-test was applied to compare the $t_{50} \%$ mean values of formulations prepared at each chitosan concentration. The calculated t-values were $2.826,1.552$ and 0.761 on applying the $t-$ test on formulations number 1 and 2 (prepared with $0.1 \% \mathrm{CS}$ ), formulations 3 and 4 (prepared with $0.25 \% \mathrm{CS}$ ) and formulations 5 and 6 (prepared with $0.4 \% \mathrm{CS}$ ), respectively.

Increasing the drug to polymer ratio from 1:1 to $1: 2$ showed a non-significant effect on the drug release from microcapsules at chitosan concentrations $0.1,0.25$ and $0.4 \%$.

The ANOVA test was applied to examine the effect of the CS concentration on the $t_{50 \%}$, where values of $\mathrm{t}_{50 \%}$ are $2.33 \pm 0.12,2.51 \pm 0.15$ and $3.08 \pm 0.19$ for formulation number 2,4 and 6 respectively. Values of $t_{50 \%}$ for formulations number 1,3 and 5 are $2.07 \pm 0.13,2.38 \pm 0.11$ and $2.67 \pm 0.17$. From table $4 \mathrm{a} \& \mathrm{~b}$ the calculated $F$ ratio was equal to 13.43 on applying the test on formulations number 1, 3 and 5 (prepared at D:P ratio of 1:1) and 17.72 on applying it on formulations number 2, 4 and 6 (prepared at $\mathrm{D}: \mathrm{P}$ ratio of 1:2). The tabulated $\mathrm{F}$ value at $\alpha=$ 0.05 and degrees of freedom of 2 and 6 (5.32), thus the calculated $F$ value exceeds the tabulated values which proves the presence of high significanct effect of CS concentration on $\mathrm{t}_{50 \%}$.

The above results revealed that the release profile of DS from alginate-chitosan coated microcapsules was dependent on the CS concentration.As the CS concentration was increased from 0.1 to $0.4 \%$, the drug release was markedly decreased. Some workers also reported that diclofenac release from its microcapsules was increased on increasing the CS concentration ${ }^{11}$. On the other hand, it was found that the drug release from alginatechitosan coated microcapsules decreased as the CS concentration in the external phase increased from 0.25 to $0.4 \%$.

The prepared microapsules employing $0.1 \%$ chitosan dissolved completely in $\mathrm{pH} 7.4$ releasing $100 \%$ of its drug content after $5 \mathrm{hrs}$. This behavior probably occurred because the CS amount was insufficient for complete complex formation between alginate and chitosan. Chitosan concentration of $0.4 \%$ was found to have the maximum retarding effect of release $^{10}$. Similarly, investigation of the characteristics of polyion complex of chitosan and alginate were carried by some workers, employing viscosity measurement, Fourier transform IR spectroscopy, elementary analysis and gel permeation chromatography. They found that the binding ratio of a unit molecule 
Table 3: ANOVA test for chitosan concentration effect on drug loading efficiency alginate-chitosan coated microcapsules (a) at D:P ratio 1:1 and (b) at D:P ratio 1:2.

(a)

\begin{tabular}{||c|c|c|c|c|}
\hline Source of variation & DF & $\begin{array}{c}\text { Sum of } \\
\text { squares }\end{array}$ & $\begin{array}{c}\text { Mean } \\
\text { squares }\end{array}$ & F ratio \\
\hline Between regimens & 2 & 0.587 & 0.264 & 0.386 \\
\hline Within regimens & 6 & 0.4252 & 0.683 & - \\
\hline Total & 8 & 0.4839 & - & - \\
\hline
\end{tabular}

(b)

\begin{tabular}{||c|c|c|c|c|}
\hline Source of variation & DF & $\begin{array}{c}\text { Sum of } \\
\text { squares }\end{array}$ & $\begin{array}{c}\text { Mean } \\
\text { squares }\end{array}$ & F ratio \\
\hline Between regimens & 2 & 0.1646 & 0.742 & 0.78 \\
\hline Within regimens & 6 & 0.6876 & 0.951 & - \\
\hline Total & 8 & 0.8522 & - & - \\
\hline
\end{tabular}

$F_{\text {tabulated }}=5.14$ at $\alpha=0.05$ and degree of Freedom $(D F), f_{1}=2$ and $f_{2}=6$

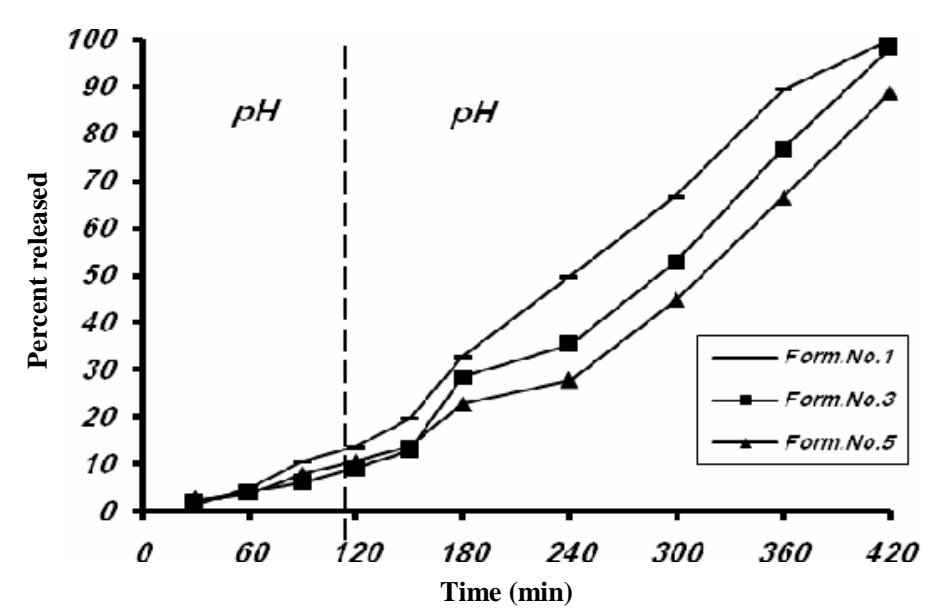

Fig. 3: Release profiles of diclofenac sodium chitosan-alginate microcapsules prepared at drug to polymer ratio of 1:1.

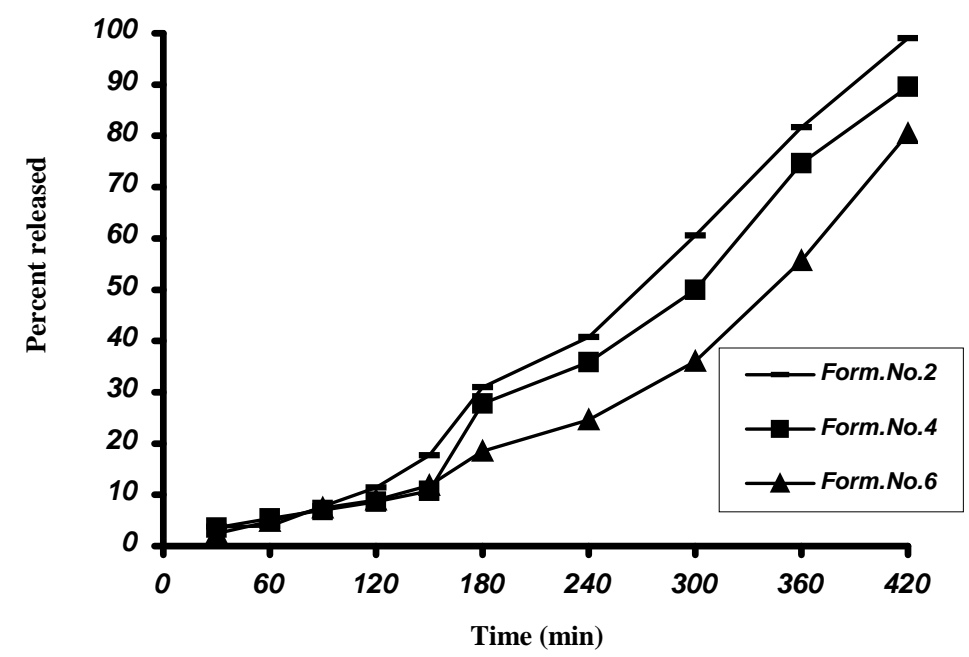

Fig. 4: Release profiles of diclofenac sodium chitosan-alginate microcapsules prepared at drug to polymer ratio of 1:2. 
of chitosan and alginae was $1: 1.5$ in weight, which was approximately equivalent to $1: 1$ of the unit molecular weight ratio and this ratio was relatively constant in the media of various $\mathrm{pH}$ values ${ }^{12}$.

The release data of diclofenac sodium from chitosan-alginate microcapsules prepared with different process variables were analyzed according to zero and first-order kinetics as well as, Higushi diffusion controlled mechanism (Table 5). The release order of the drug was determined. It was found that the drug was released from the microcapsules according to zero-order pattern, at the two drugs to polymer ratios. The release medium dissolves the drug after penetrating the microcapsule wall by allowing the dissolved drug to diffuse out due to concentration gradient established between the interior of the microcapsules and the release medium ${ }^{13}$.

Table 4: ANOVA test for chitosan concentration effect on time for 50\% of the drug to be released $\left(\mathrm{t}_{50 \%}\right)$ of alginate-chitosan coated microcapsules prepared by incubation for $6 \mathrm{~min}$. at ambient conditions: (a) At D: P ratio 1:1 and (b) At D: P ratio 1:2.

(a)

\begin{tabular}{||c|c|c|c|c||}
\hline Source of variation & DF & $\begin{array}{c}\text { Sum of } \\
\text { squares }\end{array}$ & $\begin{array}{c}\text { Mean } \\
\text { squares }\end{array}$ & F ratio \\
\hline Between regimens & 2 & 0.5411 & 0.2705 & 13.43 \\
\hline Within regimens & 6 & 0.1217 & 0.0203 & - \\
\hline Total & 8 & 0.6628 & - & - \\
\hline
\end{tabular}

(b)

\begin{tabular}{||c|c|c|c|c|}
\hline Source of variation & DF & $\begin{array}{c}\text { Sum of } \\
\text { squares }\end{array}$ & $\begin{array}{c}\text { Mean } \\
\text { squares }\end{array}$ & F ratio \\
\hline Between regimens & 2 & 0.8998 & 0.4499 & 17.72 \\
\hline Within regimens & 6 & 0.1523 & 0.0254 & - \\
\hline Total & 8 & 1.0521 & - & - \\
\hline
\end{tabular}

Ftabulated $=5.32$ at $\alpha=0.05$ and degree of Freedom $(D F), f 1=2$ and $f 2=6$

Table 5: Analysis of the release data for diclofenac sodium microcapsules prepared at drug: polymer ratios of 1:1 and 1:2 using chitosan-alginate mixture.

\begin{tabular}{|c|c|c|c|c|c|c|c|}
\hline \multicolumn{2}{|c|}{ Formula number } & $\mathrm{F} 1$ & $\mathrm{~F} 2$ & $\mathrm{~F} 3$ & $\mathrm{~F} 4$ & $\mathrm{~F} 5$ & $\mathrm{~F} 6$ \\
\hline \multirow{2}{*}{ Zero-order } & $\mathrm{K}_{\mathrm{z}}$ & 24.110 & 21.440 & 20.944 & 19.76 & 18.66 & 61.21 \\
\cline { 2 - 9 } & $\mathrm{R}_{\mathrm{z}}$ & 0.958 & $\underline{0.972}$ & $\underline{0.961}$ & $\underline{0.962}$ & $\underline{0.968}$ & 0.960 \\
\hline \multirow{2}{*}{ First-order } & $\mathrm{K}_{\mathrm{f}}$ & 0.536 & 0.581 & 0.541 & 0.447 & 0.406 & 0.301 \\
\cline { 2 - 8 } & $\mathrm{R}_{\mathrm{f}}$ & 0.893 & 0.919 & 0.904 & 0.922 & 0.903 & 0.904 \\
\hline \multirow{2}{*}{ Higuchi equation } & $\mathrm{K}_{\mathrm{h}}$ & 56.605 & 56.261 & 54.371 & 51.173 & 48.094 & 41.47 \\
\cline { 2 - 8 } & $\mathrm{R}_{\mathrm{h}}$ & 0.780 & 0.925 & 0.912 & 0.954 & 0.904 & 0.890 \\
\hline
\end{tabular}

$\mathrm{K}_{\mathrm{z}}\left(\mathrm{mg} \cdot \mathrm{hr}^{-1}\right), \mathrm{K}_{\mathrm{f}}\left(\mathrm{hr}^{-1}\right)$ and $\mathrm{K}_{\mathrm{h}}\left(\mathrm{mg} / \mathrm{cm}^{2} . \mathrm{hr}^{1 / 2}\right)$ are the release rate constants of zero-order, first order and Higuchi model kinetics, respectively, as well as $R_{z}, R_{f}$ and $R_{h}$ are their corresponding correlation coefficients. 


\section{Conclusion}

Coating the alginate microcapsules with chitosan delayed the drug release at $\mathrm{pH} 7.4$ by forming alginate-chitosan complex. The microcapsules prepared using 0.1 and $0.25 \%$ chitosan were spherical in shape while $0.4 \%$ CS formed microcapsules having rounded heads and tapered tails. The change in chitosan concentration had a non-significant effect on the particle size, the yield and the drug loading efficiency. Increasing the CS concentration from 0.1 to $0.25 \%$ decreased the drug release significantly. Chitosan coated microcapsules followed zero-order release kinetics. The behavior of the alginate-chitosan microcapsules at low $\mathrm{pH} 1.2$ and higher $\mathrm{pH} 7.4$ are of great interest for the delivery of non-steroidal antiinflammatory drug into the small intestine.

\section{REFERENCES}

1- Martindale, "The Extra Pharmacopoeia", $35^{\text {th }}$ Ed., The Pharmac. Press, London, 2007, pp. 12, 25.

2- Y. Xu, C. Zhan, L. Fan, L. Wang and H. Zheng, "Preparation of dual cross-linked alginate-chitosan blend gel beads and invitro controlled release in oral sitespecific drug delivery system", Int. J. Pharm., 336, 329 (2007).

3- B. Riva, C. Nowak, E. R. Sanchez, A. Hernández, M. Schulz-Siegmund, M. K. Pec, A. Delgado and C. Évora, "VEGFcontrolled release within a bone defect from alginate/chitosan/PLA-H scaffolds", Eur. J. Pharm. and Biopharm., 73, 50-58 (2009).

4- K. Mladenovska, O. Cruaud, P. Richomme, E. Belamie, R. S. Raicki, M.C. Venier-Julienne, E. Popovski, J. P. Benoit and K. Goracinova, "5-ASA loaded chitosan-Ca-alginate microparticles: Preparation and physicochemical characterization", Int. J. Pharm., 345, 5969 (2007).

5- R. M. Lucinda-Silva, H. R. N. Salgado and R. C. Evangelista, "Preparation of alginate/chitosan/carboxymethyl chitosan complex microcapsules and application in Lactobacillus casei ATCC393", Carb. Polym., 81, 260-268 (2010).
6- M. M. Ahmed, K. I. Saleh and A. Ismail, "Formulatin, Characterization and In-vitro Release Study of Diclofenac Sodium Microcapsles", Egyp. J. Biom. Sci., 15, 480 (2004).

7- E. M. Michal, C. P. Joseph, A. M. Maria, O. W. Robert and P. J. Keith, "Highly supersaturated solutions from dissolution of amorphous itraconazole microparticles at pH 6.8", Mol. Pharm., 6, 375 (2009).

8- A. R. de Leon and Y. Zhu, "On the number of principal components: A test of dimensionality based on measurements of similarity between matrices", Comp. Stat. and Data Anal., 52, 2228-2237 (2008).

9- L. S. Liu, S. Q. Liu, S. Y. Ng, M. Froix, T. Ohno and J. Heller, "5-ASA loaded chitosan-Ca-alginate microparticles: Preparation and physicochemical characterization", J. Cont. Rel., 43, 65 (1997).

10- S. Takka and F. Acarturk, "Calcium alginate microparticles for oral administration: I: effect of sodium alginate type on drug release and drug entrapment efficiency", J. Microencap., 16, 275 (1999).

11- M. A. Fernandez-Hervas, M. A. Holgado, A. Fini and T. Fell, "In-vitro evaluation of alginate beads of a diclofenac salt", Int. J. Pharm. 163, 23 (1998).

12- T. Takahashi, K. Takayama, Y. Machida and T. Nagai, "Characteristics of polyanion complexes of chitosan with sodium alginate and sodium polyacrylate", Adv. Drug Del. Rev., 61, 35 (1990).

13- W.-T. Kim, H. Chung, I. I.-S. Shin, K. L. Yam and D. Chung, "Characterization of calcium alginate and chitosan-treated calcium alginate gel beads entrapping allyl isothiocyanate", Carb. Polym., 71, 566 (2008). 